\title{
Triggering drug use in patients with psoriasis: an investigative report from Turkey
}

\author{
Zerrin Ogretmen ${ }^{1}$, Ulku Askin², Meliha Merve Hiz ${ }^{3}$, Sibel Cevizci ${ }^{4}$
}

\author{
${ }^{1}$ Department of Dermatology, School of Medicine, Çanakkale Onsekiz Mart University, Canakkale, Turkey \\ Head of Department: Prof. Zerrin Öğretmen MD \\ ${ }^{2}$ Department of Dermatology, Baskent University Zübeyde Hanım Training and Research Center, Izmir, Turkey \\ Head of Department: Prof. Tülin Güleç MD \\ ${ }^{3}$ Department of Biology, The Faculty of Arts and Sciences, Canakkale Onsekiz Mart University, Canakkale, Turkey \\ Head of Department: Prof. İsmet Uysal PhD \\ ${ }^{4}$ Department of Public Health, School of Medicine, Canakkale Onsekiz Mart University, Canakkale, Turkey \\ Head of Department: Assoc. Prof. Çoşkun Bakar MD
}

Postep Derm Alergol 2014; XXXI, 5: 294-298 DOI: 10.5114/pdia.2014.44019

\begin{abstract}
Introduction: The patients clinically diagnosed with psoriasis were investigated for drug use that may trigger psoriasis.

Aim: To minimize the triggering drug use and help the medical treatment of psoriasis patients.

Material and methods: The study involved 289 psoriatic patients who attended our clinic in 2010-2012 and were asked to bring their drug lists of the last year, which they obtained from the pharmacy's record system. They were advised not to use the drugs that may trigger psoriasis. Data analyses were performed using SPSS program version 19.0.

Results: A total of 289 patients were included in the study. Two hundred and twenty-one patients were using non-steroidal anti-inflammatory drugs; 133 patients were using anti-reflux drugs; 35 patients were using antidiabetic drugs; 31 patients were using calcium-channel blockers and 24 patients were using $\beta$-blockers. In our study group, there was no significantly difference between median PASI scores of the patients using a triggering drug and those of who are not using a triggering drug. However, there was a positive low correlation between PASI rates and numbers of drugs used $(r=0.180, p=0.013)$.

Conclusions: Many other factors may trigger psoriasis, therefore the effect of stopping or minimizing the drug use on disease remission is not known. Because of the high triggering drug use rate, it is important to enlighten psoriasis patients about triggering drugs.
\end{abstract}

Key words: psoriasis, triggering drugs, $\beta$-blockers, non-steroidal anti-inflammatory drugs.

\section{Introduction}

Adverse drug reactions and medication errors are major public health problems. Therefore, taking a careful medical history, superintend redundant medicine consumption and drug interaction are a critical step for prevention. There are many reports in the literature regarding the initiating, triggering and aggravated roles of drugs in psoriasis etiopathogenesis [1-8]. However, it is difficult to prove in an antidepressant receiving patient whether the stress or drug is a triggering factor. It is the same in the antibiotic use; it is unclear whether the infection or the used antibiotic is the triggering factor.
However, effects of some drugs are more prominent. Triggering effects of drugs are indicative in some drugs, like non-steroidal anti-inflammatory drugs (NSAIDs) and/or $\beta$-blockers, because lesions are recovered after the drug discontinuation. The most commonly reported drugs, which trigger psoriasis in the literature, are NSAIDs, $\beta$-blockers, synthetic antimalarial agents, lithium and tetracycline [2, 4-6].

Even biological agents, which have been reported as successful in psoriasis treatment in recent years, have also been reported that they may cause exacerbations of psoriasis lesions [8-11].

Address for correspondence: Meliha Merve Hiz PhD, Department of Biology, Faculty of Science and Arts, School of Medicine, Çanakkale Onsekiz Mart University, Terzioglu Campus, 17100, Canakkale, Turkey, fax: +90 5332444545, e-mail: mervemeliha@comu.edu.tr Received: 23.01.2014, accepted: 4.04.2014. 
Although there are no definite data related to psoriasis incidence, general consensus of dermatologists has been that the number of psoriasis cases has increased in the recent years. Socioeconomic conditions and uncontrolled drug use, which is a new trend, and also easy contamination of diseases in crowded environments are believed to be triggering factors in the psoriasis increase. It has been accepted that drugs act like exogenous antigens and they sometimes play triggering roles in psoriasis pathogenesis [2, 3, 6].

\section{Aim}

The goal of this study was to investigate a correlation between triggering drugs used in patients with psoriasis and mean PSI scores, and to draw attention to the triggering drug use in those patients.

\section{Material and methods}

Patients, who came to our outpatient clinic between 2010 and 2012 and were diagnosed with psoriasis, were requested to bring their drug lists, which were recorded in the pharmacy's computer system. After reviewing those lists, we have informed patients about the drugs that may have possibly caused the triggering. We excluded patients, who were not recorded in the Social Security System (SGK), and whose drug lists were only provided from anamnesis information, from the study. A total of 289 cases were included in the study. By using the pharmacy lists, drugs, which were used at least for 3 months and at most for 32 months were reviewed. Time intervals in the lists were related to disease durations.

\section{Statistical analysis}

Statistical analysis of data was performed using 19.0 version of the SPSS package program. Normal distribution of data was evaluated by Kolmogorov-Smirnov test.
The level of significance was accepted as $p<0.05$. Descriptive statistics were presented as mean \pm standard deviation or median (min-max) values for continuous variables. In addition, frequency $(n)$ and percentage (\%) values were used for categorical variables. It was evaluated whether there was any difference in median of PASI scores between triggering drug users and non-users in independent groups using non-parametric test (Mann-Whitney $U$ test) analysis. It was evaluated whether there was any correlation between PASI rates and numbers of drugs used using Spearman's correlation coefficient.

\section{Results}

Median age of cases was 45.00 (min: 8, max: 86) years; median age for females was 43.00 (min: 8, max: 84) years and median age for males was 47.00 ( $\mathrm{min}: 8$, max: 86) years. In this present study, 156 females (53.98\%) and 133 males (46.02\%) were included. Disease duration was $12.51 \pm 15.52$; Psoriasis Area Severity Index (PASI) value was $5.50 \pm 0.45$ (min: 0 , max: 42) points. Of 289 cases, 221 were using NSAIDs (76.5\%), and drug distribution of patients was as follows: 68 patients (30.8\%) diclofenac, 70 patients (31.7\%) ketoprofen, 37 patients (16.7\%) ibuprofen, 61 patients (27.6\%) naproxen, $35 \mathrm{pa-}$ tients (15.8\%) acetyl salicylic acid, 68 patients (30.3\%) flurbiprofen, 1 patient (0.5\%) acemetacin, and 21 patients (9.5\%) indomethacin. The list of patients, who used those drugs concomitantly, is given in Table 1.

The number of cases using $\beta$-blocker drugs was 24 (8.3\%), and the number of ACE inhibitor recipients was 59 (20.4\%). Of 31 patients using calcium channel blockers (CCB), 2 (6.5\%) were receiving diltiazem, and 9 (29.0\%) were receiving nifedipine. The number of antidepressant recipients was 105 (37\%); numbers of citalopram, fluoxetine, and trazodone recipients were 50, 9, and 5, respectively. There was no patient receiving lithium. Numbers of patients receiving drugs for diabetes and gastric

Table 1.Triggering drugs reported by patients with psoriasis

\begin{tabular}{|c|c|c|c|c|c|c|c|c|}
\hline \multirow[t]{2}{*}{ Drug } & \multicolumn{8}{|c|}{ Number of triggering drugs $(n)$} \\
\hline & Diclofenac & Ketoprofen & Ibuprofen & Naproxen & $\begin{array}{c}\text { Acetyl } \\
\text { salicylic acid }\end{array}$ & Flurbiprofen & Acemetacin & Indomethacin \\
\hline Diclofenac & 68 & 28 & 10 & 15 & 12 & 21 & 0 & 5 \\
\hline Ketoprofen & 28 & 70 & 13 & 20 & 10 & 21 & 1 & 8 \\
\hline Ibuprofen & 10 & 13 & 37 & 11 & 4 & 5 & 0 & 3 \\
\hline Naproxen & 15 & 20 & 11 & 61 & 9 & 21 & 1 & 8 \\
\hline $\begin{array}{l}\text { Acetyl salicylic } \\
\text { acid }\end{array}$ & 12 & 10 & 4 & 9 & 35 & 11 & 0 & 2 \\
\hline Flurbiprofen & 21 & 21 & 5 & 21 & 11 & 68 & 1 & 5 \\
\hline Acemetacin & 0 & 1 & 0 & 1 & 0 & 1 & 1 & 0 \\
\hline Indomethacin & 5 & 8 & 3 & 8 & 2 & 5 & 0 & 22 \\
\hline
\end{tabular}


diseases were 31 (10.7\%), and 113 (39\%) respectively. Seventeen (6\%) patients were taking terbinafine, and $40(14 \%)$ were taking corticosteroids. None of patients was receiving antimalarial drugs, whereas 16 (5.6\%) patients were receiving sulphonamide; 10 (3.5\%) were on salazopyrin and 7 (2.4\%) were on trimethoprim sulfamethoxazole. Of patients, 111 (38.4\%) were taking penicillin, and 8 (2.8\%) were taking tetracycline. The number of antidiabetic users was 31 (10.7\%). Concomitant use of NSAIDs with $\beta$-blocker agents was detected in 22 cases (7.6\%), and concomitant use of NSAIDs with antidiabetic agents was observed in 27 cases (9.3\%). Concomitant intake of NSAIDs with antibiotics was observed in 91 cases (31.5\%), whereas NSAIDs-gastric agent combination was observed in 118 cases (40.8\%).

NSAID-antibiotic- $\beta$-blocker combination was observed in 12 cases (4.2\%); NSAID-antibiotic-antidiabetic agent combination was observed in 5 cases (1.7\%); and NSAID-antibiotic- $\beta$-blocker-antidiabetic combination was used in 2 cases (0.7\%). Mean disease duration of patients was $12.5 \pm 15.5$ years.

Median value of PASI score was $5.55 \pm 0.45$ points in the total patient group. In patients, who received none of triggering drugs, the median value of PASI score was 6.15 (min: 2, max: 22) points. Median PASI score of patients, who took more than one drug was 5.50 (min: 0, max: 42 ) points $(p=0.835)$. There was a positive low correlation between PASI rates and numbers of drugs used ( $r=$ 0.180, $p=0.013)$.

\section{Discussion}

In our study, we investigated the rate of triggering drugs used by patients with psoriasis. The most com monly used drugs were NSAIDs, antibiotics and antidepressants, and gastric medications (cimetidine, famotidine, ranitidine) were following them. Antidiabetic agents, $\beta$-blockers, CCBs and ACE inhibitors, which have been increasingly used for type II diabetes and hypertension that are metabolic syndrome components related to obesity in the recent years, have also made up a significant problem in psoriasis. Because medications for hypertension and diabetes should be used permanently, their selection has become much more important. In line with the literature, although we observed that as the rate of triggering drug use was increased, the PASI rate was also increased in our study.

There are many studies, which have indicated that NSAIDs trigger psoriasis [1-8, 12, 13]. It was defined that ketoprofen (70 cases, $24.2 \%$ ), diclofenac (68 cases, $23.5 \%$ ), flurbiprofen (68 cases, $23.5 \%$ ) and naproxen (61 cases, $21.1 \%$ ) were the most commonly used NSAIDs; and they were followed by ibuprofen (37 cases, 12.8\%), acetyl salicylic acid (35 cases) and indomethacin (22 cases) in our study. Moreover, there were many patients, who were taking one or more of those drugs together. Median value of PASI score was $5.55 \pm 0.45$ points in the total patient group. In patients, who received none of triggering drugs, the median value of PASI score was 6.15 (min: 2, max: 22) points. Median PASI score of patients, who took more than one drug was 5.50 (min: 0, max: 42) points. There was no statistically significant difference in median PASI scores between triggering drug users and non-users.

$\beta$-Blockers have been frequently reported as triggering drugs for psoriasis in the literature [14-18]. $\beta$-Blocker use was observed in 26 of our patients. ACE inhibitors have also been reported as triggering drugs for psoriasis [19-22]. Our 63 patients (22\%) were receiving ACE inhibitors. In our study group, 14 patients (4.9\%) were receiving the ACE inhibitor- $\beta$-blocker combination.

It was reported in previous studies that also calcium channel blockers triggered psoriasis [23-26]. In our study, 31 patients (11\%) were taking calcium channel blockers. Seven of our patients (2.4\%) were taking CCB-ACE inhibitor combination, whereas 7 patients (2.4\%) were taking CCB- $\beta$-blocker combination.

Stress factor is important as an initiator and booster for lesions. Antipsychotic drug use has been increasing recently because of stress [27-33]. In the literature, it has been indicated that activation of psoriasis lesions has been caused especially by lithium use [27-29]. In our study, there was no lithium receiving patient, but antidepressant distribution among the cases was as follows: citalopram $=50(17.3 \%)$, fluoxetine $=9(3.1 \%)$ and trazodone $=5$ (1.7\%).

Also antibiotics are one of uncontrollably used drug groups. There are publications about their triggering roles of psoriasis. Especially commonly used penicillin [34], tetracycline [35] and sulfonamides [36] have been reported as triggers. In our study, 111 patients (38.4\%) were using penicillin, whereas 8 patients (2.8\%) were taking tetracycline. Among sulfonamide using 16 patients (5.6\%), 9 were taking salazopyrin and 7 were taking trimethoprim sulfamethoxazole. However, because those drugs were used for a short time and infections were triggers for psoriasis, drugs which were used for infection treatments were not mentioned as frequently as analgesics, antihypertensive and antidiabetic agents in the literature [1-8].

In the literature, it was reported that antidiabetic drugs could also trigger psoriasis [37-40]. In our study, the number of patients on antidiabetic agents was 35 (12\%), and other drugs, which our patients used and were known as triggers, were terbinafine [41-43] and antimalarial drugs [1-8]. Terbinafine was used by 17 cases (6\%). There was no patient on the antimalarial agents. There are contradictory publications in the literature about gastric medications. Although there have been publications, which indicated that they could cause psoriasis-like skin rash [44], there were also publications, which indicated that they were not triggers, even they could be used in the treatment [45-48]. In our study, 
the number of patients on gastric medications was 113 (39\%).

It has also been reported that alcohol and its metabolites might be triggering skin lesions of psoriasis [49, 50]. Moreover, as alcohol use increases liver toxicities of systemic antipsoriasis drugs, patients should be warned about alcohol use. In our study, we asked about alcohol drinking habits of patients, and informed them about its triggering effect. In our study, 86 cases (31\%) were taking alcohol. Two of our cases were hospitalized and received treatment for drinking alcohol, and it was observed that numbers of their lesions were decreased.

As concomitance of psoriasis with metabolic syndrome is a known fact, patients should also be warned about obesity. Health problems, which appear along with obesity, and drugs used for those conditions (antihypertensives, antidiabetics) complicate struggling against the disease.

The aim of this present study was not to define which drugs triggered the disease, but to direct attention of patients with psoriasis to possible triggers. Moreover, healthcare personnel also have important responsibilities. Existence of psoriasis should be definitely reviewed and investigated before recommending or prescribing especially a triggering drug. In the literature it was reported that roles of triggering drugs could be encountered not only after the first use but months after the use, so that drug effect could be missed [8, 9, 23-25, 30]. Even after treatment with biological agents, drug effects, which might increase lesions, might be observed months after the use $[8,9]$. Increased PASI score in patients with many drugs use has indicated roles of drugs. Multiple drug use and high PASI scores of advanced age patients (over 50 years) may be an indicator.

In our study group, there was no significantly difference between median PASI scores of the patients using a triggering drug and those of whom are not using a triggering drug. This result in our study may be caused by taking into consideration only drugs reported as triggering in the literature not the others. Some drugs which are used by our patients and not reported as triggering before in the literature may have been triggering. In addition, it is known that there have been several triggering factors such as alcohol consumption, smoking, infections, stress other than drugs in the patients with psoriasis. Therefore, in order to determine triggering roles of the drugs, during statistical analysis, PASI scores of the patients who have no triggering factor should be compared. On the other hand, it seems that it is not possible to find enough cases in practice. However, we found that there was a positive low correlation between PASI rates and numbers of drugs used $(r=0.180, p=0.013)$.

\section{Conclusions}

Treating psoriasis patients, who are receiving psoriasis triggering drugs (77\% NSAIDs, 8.3\% $\beta$-blocker, 37\% anti- depressants), is similarly difficult as treating a subject with the strawberry allergy using only antihistaminics.

According to pharmacogenetic studies, the same drug may not show the same effect in all subjects, but careful use of drugs and increasing awareness of patients against the disease are important. Thus, we believe that we can be more helpful to our patients. If we emphasize that the drugs they are using can also trigger their diseases and we provide that they are more careful about the issue, then we may also reveal drugs, which are not on the list yet. Physicians working at the primary healthcare settings should be warned about this issue. We believe that especially a warning in the patient leaflet sheet, which underlines that $\beta$-blockers should be carefully used in subjects with psoriasis, is not being read by patients.

Currently, we observe that many psoriasis patients accept their diseases with some localized plaques on their bodies, and they continue living by using topical preparations from time to time. We can inform our patients about triggering factors in a very limited time at the outpatient clinic visits with the help of a newsletter or a copy of the text about drugs prepared by the dermatology association. Sunny climate and thermal springs of our country as well as easily and cheaply reached seafood facilities provide conditions helpful for psoriasis treatment. We can be more helpful to our patients by using protective, rehabilitating and life quality increasing methods like group therapies instead of antidepressants, struggling against obesity, and using drugs from other groups rather than psoriasis triggering ones. We believe that another important topic is that healthcare trainings should be given to parents with psoriasis how to protect their children (with genetic susceptibility background) from triggering drugs.

\section{Acknowledgments}

This study was conducted in the Dermatology Department of Training and Research Hospital of Canakkale Onsekiz Mart University.

\section{References}

1. Kurt R, Ruocco V. Psoriasis triggered. Illan Exp Med Biol 1999; 455: 221-5.

2. Basavaraj $\mathrm{KH}$, Ashok NM, Rashmi R, et al. The role of drugs in the induction and/or exacerbation of psoriasis. Int J Dermatol 2010; 49: 1351-61.

3. Rongioletti F, Fiorucci, Parodi A. Psoriasis induced or aggravated by drugs. J Rheumatol 2009; 83: 59-61.

4. Fry L, Baker BS. Triggering psoriasis: the role of infections and medications. Clin Dermatol 2007; 25: 606-15.

5. Tsankov N, Angelova ben Kazandjieva J. Drug-induced psoriasis. Recognition and management. J Clin Dermatol Am 2000; 1: 159-65.

6. Abel EA. Diagnosis of drug-induced psoriasis. Semin Dermatol 1992; 11: 269-74. 
7. Kim GK, Del Rosso JQ. Drug provoked psoriasis. Is it drug induced or drug aggravated understanding pathophysiology and clinical relevance. J Clin Aesthet Dermatol 2010; 3: 32-8.

8. Borrás-Blasco J, Gracia-Perez A, Nuńez-Cornejo C, et al. Exacerbation of psoriatic skin lesions in a patient with psoriatic arthritis receiving adalimumab. J Clin Pharm Ther 2008; 33: 321-5.

9. Ko JM, Gottlieb AB, Kerbleski JF. Induction and exacerbation of psoriasis with TNF-blockade therapy: a review and analysis of 127 cases. J Dermatolog Treat 2009; 20: 100-8.

10. Sanso Sureda A, Rocamora Duran V, Sapina Camaro A, et al. Ustekinumab in a patient with Crohn's disease and anti-TNF-alfa-induced psoriasis. Gastroenterol Hepatol 2011; 34: 546-50.

11. Mourao AF, Rustin M, Isenberg D. Exacerbation of psoriatic skin lesions in patients with psoriatic arthritis receiving anti-tumor necrosis factor-alpha therapy: description of 3 cases and review of the literature. Clin Exp Rheumatol 2010; 28: 408-10.

12. Abel EA, DiCicco LM, Orenberg EK, et al. Drugs in exacer bation of psoriasis. J Am Acad Dermatol 1986; 15: 1007-22.

13. Ben-Chetrit E, Rubinow A. Exacerbation of psoriasis by ibuprofen. Cutis 1986; 38: 45.

14. Van Dam D, Bosch TM, Mookhoek EJ, et al. Treatment of clozapine-induced tachycardia with propranolol complicated by psoriasis. Tijdschr Psychiatr 2012; 54: 285-9.

15. Waqar S, Sarkar PK. Exacerbation of psoriasis with betablocker therapy. CMAJ 2009; 181: 60

16. O'Brien M, Koo J. The mechanism of lithium and beta-blocking agents in inducing and exacerbating psoriasis. J Drugs Dermatol 2006; 5: 426-32.

17. Arntzen N, Kavli G, Volden G. Psoriasis provoked by beta-blocking agents. Acta Derm Venereol 1984; 64: 346-8.

18. Halevy S, Feuerman EJ. Psoriasiform eruption induced by propranolol. Cutis 1979; 24: 95-8.

19. Coulter DM, Pillans PI. Angiotensin-converting enzyme in hibitors and psoriasis. N Z Med J 1993; 106: 392-3.

20. Tamir A, Wolf R, Brenner S. Exacerbation and induction of psoriasis by angiotensin-converting enzyme inhibitors. J Am Acad Dermatol 1994; 30: 1045.

21. Wolf R, Ruocco V. Triggered psoriasis. Adv Exp Med Bio 1999; 455: 221-5.

22. Lamba G, Palaniswamy C, Singh T, et al. Psoriasis induced by losartan therapy: a case report and review of the literature. Am J Ther 2011; 18: e78-80.

23. Cohen AD, Kagen M, Friger M, et al. Calcium channel blockers intake and psoriasis: a case-control study. Acta Derm Venereol 2001; 81: 347-9.

24. Nakayama J, Koga T, Furue M. Long-term efficacy and ad verse event of nifedipine sustained-release tablets for cyclosporin A-induced hypertension in patients with psoriasis. Eur J Dermatol 1998; 8: 563-8.

25. Kitamura K, Kanasashi M, Suga C, et al. Cutoneous reactions induced by calcium channel blocker: high frequency of psoriasiform eruptions. J Dermatol 1993; 20: 279-86.

26. Jafferany $M$. Lithium and psoriasis: what primary care and family physicians should know. Prim Care Companion J Clin Psychiatry 2008; 10: 435-9.

27. Brauchli YB, Jick SS, Curtin F, et al. Lithium, antipsychotics, and risk of psoriasis. J Clin Psychopharmacol 2009; 29. 134-40.

28. Allan SJ, Kavanagh GM, Herd RM, et al. The effect of inositol supplements on the psoriasis of patients taking lithium: a randomized, placebo-controlled trial. Br J Dermatol 2004; 150: 966-9

29. Lozano García MC, Baca García E. Psoriasis and lithium treatment: a common physiopathology. Actas Esp Psiquiatr 2002; 30: 400-3.

30. Hemlock C, Rosenthal JS, Winston A. Fluoxetine-induced psoriasis. Ann Pharmacother 1992; 26: 211-2.

31. Latini A, Carducci M. Psoriasis during therapy with olanzapine. Eur J Dermatol 2003; 13: 404-5.

32. Dorevitch A, Cohen-Adad G, Jabotinsky-Rubin K, et al. Lithium-associated psoriasis. Harefuah 1994; 127: 228-9.

33. Tan Pei Lin L, Kwek SK. Onset of psoriasis during therapy with fluoxetine. Gen Hosp Psychiatry 2010; 32: 446.e9-10.

34. Katz M, Seidenbaum M, Weinrauch L. Penicilin-induced generalized pustuler psoriasis. J Am Acad Dermatol 1987; 17: 918-20.

35. Tsankov N, Botev-Zlatkov N, Lazarova AZ, et al. Psoriasis and grugs: influence of tetracyclines on the course of psoriasis. J Am Acad Dermatol 1988; 19: 629-32.

36. Bliddal H, Stangerup M. Psoriasis-like skin reaction in a patient with rheumatoid arthritis after sulphasalazine therapy. Clin Rheumatol 1991; 10: 178-80.

37. Mas-Vidal A, Santos-Juanes J, Esteve-Martinez A, et al. Psoriasiform eruption triggered by a dipeptidyl peptidase IV inhibitor. Australas J Dermatol 2012; 53: 70-2.

38. Hogan AE, Tobin AM, Ahern T, et al. Glukagon-like peptide-1 (GLP-1) and the regulation of human invariant natural killer T cells: lessons from obesity, diabetes and psoriasis. Diabetologia 2011; 54: 2745-54.

39. Koca R, Altınyazar HC, Yenidünya S, et al. Psoriasiform drug eruption associated with metformin hydrochloride: a case report. Dermatol Online J 2003; 9: 11.

40. Fisher DA, Elias PM, LeBoit PL. Exacerbation of psoriasis by the hypolipidemic agent, gemfibrozil. Arch Dermatol 1988; 124: 854-5.

41. Le Guyadec T, Saint-Blancard P, Bosonnet S, et al. Oral terbinafine-induced plantar pustular psoriasis. Ann Dermatol Venereol 2000; 127: 279-81.

42. Szepietowski JC. Terbinafine exacerbates psoriasis: case report with a literature review. Acta Dermatovenerol Croat 2003; 11: 17-21.

43. Gupta AK, Sibbald RG, Knowles SR, et al. Terbinafine therapy may be associated with the development of psoriasis de novo or its exacerbation: four case reports and a review of drug-induced psoriasis. J Am Acad Dermatol 1997; 36 : 858-62.

44. Fetil E, ilknur T, Birgin B, et al. Drug induced psoriasis. Turkderm 2002; 36: 105-9.

45. Zonneveld IM, Meinardi MM, Karlsmark T, et al. Ranitidine does not affect psoriasis: a multicenter, double-blind, placebo-controlled study. J Am Acad Dermatol 1997; 36: 932-4.

46. Nielsen HJ, Kristensen JK, Hansen U, et al. Clinical effect of ranitidine in psoriasis. An open prospective study. Ugeskr Laeger 1997; 159: 598-600.

47. Wallach D, Cottenot F. Cimetidine versus placebo in the treatment of psoriasis. Dermatologica 1982; 165: 197-203.

48. Andersen M. Exacerbation of psoriasis during treatment with $\mathrm{H} 2$ antagonists. Ugeskr Laeger 1991; 153: 132.

49. Kazakevich N, Moody MN, Landau JM, et al. Alcohol and skin disorders: with a focus on psoriasis. Skin Therapy Lett 2011; 16: $5-6$

50. Bordignon M, Viel G, Peserico A, et al. Non-alcoholic fatty liver disease, alcohol intake and psoriasis. J Hepatol 2010; 53: 587. 